\title{
La participación escolar de las familias a través de plataformas digitales ${ }_{1}$
}

\author{
The School Participation of Families Through Digital Platforms
}

\author{
$\mathrm{M}^{\mathrm{a}}$ José Waliño-Guerrero \\ Maria.Jose.Walino@uv.es \\ Universitat de València \\ M. Isabel Pardo Baldoví \\ Misabel.Pardo@uv.es \\ Universitat de València \\ Graciela Esnaola Horacek \\ graesnaola@gmail.com \\ Universidad Nacional de Tres de Febrero (Argentina) \\ Ángel San Martín Alonso \\ Angel.Sanmartin@uv.es \\ Universitat de València
}

Fecha presentación: 09/03/2018 | Aceptación: 15/05/2018 |Publicación: 22/06/2018

\section{Resumen}

La educación democrática y participativa se reivindica desde hace varias décadas. La aplicación de las tecnologías digitales en la gestión de las instituciones ha contribuido a redefinir el concepto de participación. Dicho concepto se enraíza en las prácticas culturales de las familias, por ello, nos hemos detenido en la percepción que del modelo emergente de participación evidencian una muestra de madres y padres de alumnos en el ámbito de la Comunitat Valenciana. Focalizamos la atención sobre el equipamiento y la conectividad de los hogares, requisitos imprescindibles para interactuar con las plataformas. A continuación, analizamos las representaciones que las familias nos explicitan mediante entrevistas semiestructuradas y un grupo de discusión. Descubrimos que el equipamiento orienta la participación más hacia las dimensiones relativas al control que a la colegialidad en la toma de decisiones. Es necesario seguir profundizando sobre cómo está redefiniéndose la participación en un sentido más instrumental que político.

Palabras clave: participación familiar; organización escolar; tecnología educativa; alfabetización digital

\section{Resum}

L'educació democràtica i participativa es reivindica des de fa diverses dècades. L'aplicació de les tecnologies digitals en la gestió de les institucions ha contribuït a redefinir el concepte de participació. Aquest concepte s'arrela en les pràctiques culturals de les famílies i, per això ens hem detingut en la percepció que del model emergent de participació evidencien una mostra de mares i pares d'alumnes en l'àmbit de la Comunitat Valenciana. Focalitzem l'atenció sobre l'equipament i la connectivitat de les llars, requisits imprescindibles per a interactuar amb les plataformes. A continuació, analitzem les representacions que les famílies ens expliciten a través d'entrevistes semiestructurades i un grup de discussió. Descobrim que l'equipament, orienta la participació més cap a les dimensions relatives al control que a la collegialitat en la presa de decisions. És necessari seguir aprofundint sobre com s'està redefinint la participació en un sentit més instrumental que polític.

Paraules clau: participació familiar; organització escolar; tecnologia educativa; alfabetització digital

\footnotetext{
${ }^{1}$ Gran parte de los datos y referencias de este artículo se han obtenido en el marco del proyecto "La escuela de la sociedad digital: análisis y propuestas para la producción y uso de los contenidos digitales educativos" (Ref. EDU2015-64593-R). Financiado por el Programa Estatal de I+D+i Orientada a los Retos de la Sociedad convocado por el Gobierno de España.
} 
Waliño-Guerrero, Ma José; Pardo Baldoví, M. Isabel; Esnaola Horacek, Graciela y San Martín Alonso, Ángel (2018). "La participación escolar de las familias a través de plataformas digitales". @tic revista d'innovació educativa, 20, 80-88.

\begin{abstract}
A democratic and participative education has been claimed for several decades. The application of digital technologies in the management of institutions has helped to redefine the concept of participation. This concept is rooted in the cultural practices of families and, for this reason, we have analyse the perception of the emerging participation model evidenced by a sample of families of students in the area of the Valencian Community. We focus attention on the equipment and connectivity of homes, both are essential requirements to interact with the platforms. Next, we analyse the representations that families make explicit to us through semi-structured interviews and a focus group. We discover that technological equipment directs participation more towards the dimensions related to control than to collegiality in decision-making. It is necessary to continue deepening in the ways of how participation is being redefined in a more instrumental than in a political sense.
\end{abstract}

Key Words: parent participation; school organization; educational technology; digital literacy

Desmercantilizar es un imperativo indispensable en la búsqueda de una sociedad mejor.

\section{B. De Sousa Santos}

\section{Escenas de participación}

Hace unos días terminó un talent show televisivo decidiendo el triunfo a golpe de clic. Durante el popular concurso, un grupo de jóvenes aprenden las artes de la canción ligera. La ganadora fue elegida, en gran medida, mediante el voto de la audiencia emitido a través de los dispositivos móviles. Al día siguiente, en la entradilla del informativo de la cadena promotora, se afirmaba que la ganadora había sido elegida participativa y democráticamente. Casi cuatro millones de telespectadores habían seguido la gala final, en el transcurso de la cual emitieron su voto en favor de uno de los cinco finalistas. Entre la televisión y las redes sociales, convirtieron el concurso en un verdadero fenómeno de masas. Pero este hecho, entendemos, no convierte al citado procedimiento de votación en un proceso cabal de participación.

Unos días más tarde, asistimos a un acto académico con mucho público y bastantes atractivos. El conferenciante puso en marcha, de pronto, un Kahoot con el que pretendía visibilizar los estereotipos que los asistentes teníamos sobre el profesorado. El ponente, renombrado profesor universitario, fue planteando una serie de cuestiones para contestar desde nuestro teléfono móvil. La respuesta era elegir una de las 4 alternativas que se nos ofrecía en la pantalla. La citada aplicación es gratuita y permite crear concursos de pregunta-respuesta haciendo luego un tratamiento básico de datos. La aplicación se nos presenta como una herramienta orientada a fomentar la participación de los estudiantes en la construcción del conocimiento. Sin duda, ese primer objetivo lo consiguió pues el ponente mostró que en torno al $90 \%$ de los asistentes había participado, de los más de 200 asistentes, en su mayoría estudiantes universitarios.

Pocos días después tuvo lugar la convocatoria de elecciones de rector o rectora de la universidad en la que se celebró la citada conferencia. Pues bien, en la facultad a la que pertenecen los estudiantes tan unánimes ante el Kahoot, el porcentaje de quienes ejercieron su derecho de emitir un voto en las urnas, apenas llegó al 4\%. Es obvio que hay muchas razones para explicar este fenómeno, pero no deja de ser Ilamativa la desigual adhesión suscitada por una y otra convocatoria. Lo que parece apuntar que, tanto a nivel social como académico, se está produciendo un cambio en los formatos en los que se ejerce la participación y en los aspectos que generan su interés y la promueven. ¿Este fenómeno podría ser concomitante con el observado en las enseñanzas no universitarias?

La referencia a estas dos situaciones acaecidas con muy poca diferencia de tiempo, nos invita a plantear algunas cuestiones: ¿las soluciones tecnológicas orientadas a fomentar el edutainment son igualmente valiosas en los procesos organizativos de los centros? ¿Estamos asistiendo a una redefinición de las formas mediante las que se ejerce la participación escolar? ¿Qué tipo de participación, y con qué legitimidad, de las familias favorecen las plataformas digitales? ¿Hemos de formar a familias y estudiantes en las políticas y prácticas de la participación a través de plataformas digitales?

\section{La participación: componente estratégico de las organizaciones escolares}

La necesidad de construir una cultura escolar democrática y participativa es una demanda recurrente en nuestra historia escolar reciente. Participación que alude tanto a profesorado y alumnado como a las familias, abogando por un concepto amplio que fomente la consolidación de la escuela como comunidad educativa. En esta línea, la participación de los distintos agentes escolares en la educación no solo se considera algo fundamental para la correcta formación de las futuras generaciones, sino que también es un derecho constitucional (art. 27.5 de la Constitución Española de 1978) que ha sido recogido con mayor o menor acierto y proporción en las sucesivas modificaciones legislativas. Planteamiento retomado a partir de las nuevas configuraciones sociales del siglo XXI reafirmadas por la cultura 2.0 según los informes Horizon 2017 (Freeman et al., 2017) y para otros estudiosos se circunscribe a los derechos individuales (Feito, 2014).

No obstante, que el plano discursivo visibilice las demandas de participación de las familias en la escuela no implica que esto se traslade al plano real de la vida en los centros. Más todavía en el contexto actual, en el que la progresiva generalización de las políticas y prácticas inspiradas en el pensamiento neoliberal, está provocando la reestructuración de las instituciones, entre ellas la escolar.

Si reflexionamos sobre la tradición de la participación en la escuela apreciaremos que esta no siempre ha tenido incorporadas en sus estructuras los procesos participativos. La institución escolar, gestada en la modernidad, lleva la impronta de prácticas sociales ancladas en lógicas de poder burocrático y vertical, donde, además, el rol del docente y del estudiante 
Waliño-Guerrero, Ma José; Pardo Baldoví, M. Isabel; Esnaola Horacek, Graciela y San Martín Alonso, Ángel (2018). "La participación escolar de las familias a través de plataformas digitales". @tic revista d'innovació educativa, 20, 80-88.

estaban claramente diferenciados. El diálogo y el consenso, en la participación de todos los actores, rozaba los bordes del caos disciplinario.

Sin embargo, y sin remontarnos demasiado atrás, durante el último cuarto del siglo XX, las cuestiones asociadas a la participación formaron parte central de las reivindicaciones de los movimientos sociales y políticos. La sociedad en general estaba removiendo sus bases hacia lógicas más participativas, dándole al ciudadano un rol mucho más activo. Las redes sociales y las comunidades organizadas contribuyeron a crear un nuevo escenario. En congruencia con estos cambios socioculturales, se empezó a reclamar la incorporación de prácticas participativas hasta el momento ausentes en el funcionamiento regular de las instituciones educativas. A partir de ese momento se comenzaron a contemplar el diálogo, la crítica y hasta la corresponsabilidad en los procesos de toma de decisiones. Se consideraba fundamental el que, como señalaba Segovia (1986), la gestión democrática de los centros comenzara a dar cabida a la pluralidad de valores sociales.

En esta misma línea de pensamiento Gómez (2000), afirma que la sociedad liberal llevó la libertad de pensamiento a la escuela pública, por lo cual pide que ahora la sociedad democrática aporte "no solo la universalización del servicio, sino su prestación en forma participativa; otorgando a los miembros de la comunidad escolar el derecho a ser copartícipes en la ordenación de su trabajo" (p. 80). Este profesor y parlamentario de las primeras legislaturas, fue uno de los artífices del modelo de participación de nuestro sistema escolar regulado en la LODE de 1985 y que las sucesivas leyes orgánicas fueron desfigurando progresivamente.

Para muchos autores la participación es el mejor medio para implicar a los distintos agentes escolares, esto no solo evita la despolitización de aquellos, sino que además a partir del conocimiento que adquieren de los centros escolares han de tomar parte en las tareas de decisión. Proceso que, a su vez, contribuye a limar la excesiva burocratización que envuelve a las instituciones educativas según Bates (1989). Asimismo, la participación genera comunidad, estructuras fuertes y de inserción de la escuela en el entorno social, económico y cultural mediante el uso de distintas estrategias de negociación, resolución de problemas e intercambio de información (Vanvergen, 1979).

Tras todo lo expuesto es necesario asumir que la participación conlleva una particular implicación política, entre otros motivos porque es uno de los medios para hacer y expresar la libertad de los individuos en el espacio público. Para la filósofa Hanna Arendt (1996), la política y la libertad son las dos caras de una misma moneda, hasta el punto que la una no es posible sin la otra. Ahora bien, es necesario un espacio público común para que puedan expresarse mediante el pensamiento y la acción. Por eso la citada filósofa sostiene que un mundo organizado políticamente es aquel en el que mujeres y hombres libres pueden insertarse de palabra y obra.

Por ello, la reivindicada participación en la escuela se considera condición necesaria para atender la dimensión pública de la educación, la que hace que sea del común $y$ que todas $y$ todos tengan iguales derechos $y$ oportunidades. Para alcanzar este propósito político, resulta imprescindible la participación individual y colectiva de todos los agentes sociales con algún interés en la educación. Constatación que pone en primer plano las cuestiones relacionadas con el ejercicio del poder y, por tanto, al posicionamiento ideológico de los distintos colectivos de la comunidad escolar.

\section{Reestructuración de la participación}

Bajo este epígrafe queremos centrar nuestro objeto de estudio y plantear algunas de las reflexiones que nos suscita la apertura semántica experimentada por el término de participación al someterse a la lógica de alguna suerte de tecnología. En esta cuestión nos remitimos a los distintos trabajos de autores como Boudon (1983), Mattelart y Vitalis (2015) o Sartori (1998), entre otros. Richard Sennett (2000) explica que la reingeniería o reestructuración experimentada por las organizaciones, se produce cuando incorporan a su funcionamiento cotidiano las tecnologías, la computación y las redes. Entre otros cambios se experimenta una desagregación vertical de sucesivas capas de la estructura, pero sin que se produzca una verdadera descentralización del poder.

La desconcentración mediante las redes tecnológicas no fomenta una mayor participación democrática, ya que esta exige deliberación y discusión cara a cara. En un sentido muy parecido se pronuncian autores como Maxcy (1995) o Shields (1996). Cuando Subirats (2016) analiza los distintos modelos de gobernanza basados en las tecnologías de la información, mantiene que la estrategia supone aminorar la capacidad de influencia en las instituciones de los poderes públicos y también de la ciudadanía.

Pero esta tendencia es imparable porque es uno de los principios del hegemónico neoliberalismo frente a las organizaciones (Ball, 2016). De modo que la cuestión fundamental deberá ser cómo afrontar a partir de las distintas modalidades de participación la emergente reestructuración de las organizaciones, y en nuestro caso de las educativas en las que tanto se usan las redes sociales bajo la impronta de la participación (Zhao et al., 2008).

Una referencia obligada en este proceso de transformación es la expresión de cultura participativa acuñada por Jenkins (2008). Más que hablar de productores y consumidores mediáticos como si desempeñasen roles separados, propone considerarlos hoy como participantes que interactúan conforme a un nuevo conjunto de reglas que ninguno de nosotros comprende de modo cabal. En cualquier caso, las formas tradicionales de participación se suplantan por otras más distribuidas e igualitarias apoyadas en la expresividad espontánea de los individuos (Aparici y Osuna, 2013; Helsper y Eynon, 2010; Inglehart y Welzel, 2006).

Sobre este particular, resulta muy interesante la observación de Innerarity (2011) cuando mantiene que la única utopía que sigue viva es la de la desintermediación. Por su parte, Benkler (2015) advierte del valor constituyente de las sociedades democráticas de las instancias de intermediación, por lo que su desaparición provocará un cambio radical en la estructura de las organizaciones. En definitiva, la que defiende que ningún intermediario es necesario ni despierta ya confianza alguna.

\section{Marco metodológico}

A partir de lo expuesto en los apartados precedentes, el problema que convoca el presente estudio se centra en analizar las valoraciones y percepciones de uno de los 
Waliño-Guerrero, Ma José; Pardo Baldoví, M. Isabel; Esnaola Horacek, Graciela y San Martín Alonso, Ángel (2018). "La participación escolar de las familias a través de plataformas digitales". @tic revista d'innovació educativa, 20, 80-88.

agentes que intervienen en la organización y gestión de los centros escolares, como son las familias. Estudiamos, en particular, las tareas que realizan en las plataformas puestas a disposición de las instituciones educativas por la Administración y otras muchas empresas, como las editoriales. En definitiva, nos interesa conocer las percepciones y valoraciones que sobre la participación realizan las familias en un ámbito, como la educación de sus hijos e hijas, que requiere tanta implicación.

Para alcanzar este propósito hemos procedido en dos direcciones. La primera ha sido analizar estudios e informes solventes sobre el equipamiento de los hogares valencianos, por considerarlo un requisito imprescindible para el ejercicio de la participación a través de plataformas. Se han analizado informes promovidos desde instancias europeas, estatales y autonómicas. A partir de estos datos de naturaleza cuantitativa, nos acercarnos a los agentes escolares objeto de estudio a fin de indagar sobre sus percepciones y apreciaciones valorativas desde un enfoque cualitativo. Para ello hemos seguido las aportaciones de orden metodológico de autores como Gill (2011), Pereira (2011) o Stake (2010), y en particular las de Smith et al. (2011) por la coincidencia de planteamiento y resultados.

Siguiendo el protocolo del proyecto competitivo "La escuela de la sociedad digital: análisis y propuestas para la producción y uso de los contenidos digitales educativos" (Ref. EDU2015-64593-R), financiado por el Programa Estatal de I+D+i Orientada a los Retos de la Sociedad convocado por el Gobierno de España, en el que se inscribe este trabajo, realizamos entrevistas semiestructuradas a miembros de las familias con hijos e hijas en $5^{\circ}$ y $6^{\circ}$ curso de Primaria. Todos pertenecían a 4 centros públicos y 1 concertado, seleccionados por trabajar en la inclusión activa de las TIC y manejar materiales didácticos digitales. Se realizaron un total de 20 entrevistas (14 mujeres y 6 hombres) y un grupo de discusión con quienes pudieron asistir el día de la convocatoria (5 de la pública y 2 de la concertada). Las sesiones debían durar entre 45 y 60 minutos, el guion se les facilitó, así como el impreso de autorización para la grabación sonora y posterior transcripción. Los documentos con los relatos transcritos se les facilitaron a los entrevistados, si bien casi nadie propuso modificaciones o aclaraciones a lo transcrito.

Posteriormente, con el programa Atlas-Ti 8, efectuamos un primer nivel de análisis orientado a cuantificar la acumulación de frecuencias en torno a las categorías preseleccionadas. En un segundo momento tratamos de identificar las redes relacionales en las que se sustentan los relatos construidos por los informantes. De modo que tanto el análisis de las entrevistas como del grupo focal se hizo a partir de una serie de categorías que consideramos indicadores clave para identificar las estrategias, valoraciones y percepciones de las familias en torno a la participación. Las categorías preseleccionadas surgieron a partir del marco conceptual precedente y del cotejo de trabajos como los de Smith et al. (2011) y Macià (2016), entre otros.

\section{Discusión de los resultados más relevantes}

Tras la exposición del marco teórico y metodológico, pasamos a la exposición y discusión de los resultados más significativos según el propósito que orienta este trabajo. En primer lugar, revisamos los datos relativos a la accesibilidad y la conectividad, en tanto que requisito y determinante del problema estudiado aquí; mientras que en el segundo paso nos ocupamos propiamente de los relatos que los sujetos nos facilitaron sobre su experiencia de participación en la vida de centro.

\subsection{Cobertura tecnológica}

Tal como hemos expuesto en párrafos anteriores, el equipamiento tecnológico y la conectividad de los hogares resultan condiciones indispensables para ejercer el tipo de participación analizado en este trabajo. Y desde esta perspectiva, resulta particularmente interesante el dato evolutivo de la conectividad española, que ha experimentado un crecimiento espectacular en los últimos años. Concretamente, durante el período 2012-2016, España fue el país de la OCDE con mayor crecimiento de la fibra óptica en sus hogares, hasta el punto de ocupar el cuarto lugar en este tipo de equipamiento (Telefónica, 2018). Por lo que se refiere al sistema escolar, la mejora de conectividad responde a que bajo la tutela de la Estrategia Europa2020 y con fondos europeos se adoptan dos iniciativas muy relevantes: se concentra en RedIRIS todas las redes educativas, desde educación infantil hasta universidad y, segundo, se propone que en 2020 todos los estudiantes de la educación pública dispongan en sus centros de banda ancha ultrarrápida (VV.AA, 2015).

Volviendo la mirada a los hogares, en la gráfica de la Figura 1 se aprecia el elevado porcentaje de conexión de banda ancha en los hogares españoles, especialmente a través de conexiones de ADSL y ahora también de fibra óptica. Como puede observarse en la figura citada, y según los datos de INE (2016), actualmente la conectividad a través de ADSL (48'3\%) es todavía mucho mayor que la de fibra óptica (37'5\%). No obstante, la tendencia emergente es hacia el crecimiento de la fibra óptica en detrimento del ADSL. En cualquier caso, si consideramos que es en los hogares donde mayormente se conectan para realizar diferentes tareas, incluidas las escolares, el gasto de los hogares deviene en indicador de la calidad de los procesos de enseñanza y aprendizaje (Pérez y Uriel, 2016).

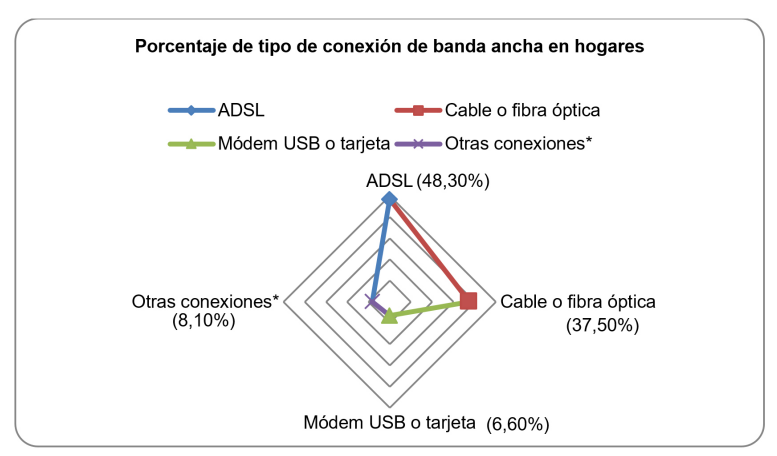

*Otras conexiones de banda ancha (vía satélite, Wi-Fi público o WiMAX)

Figura 1. Gráfico de la distribución del tipo de conexión de banda ancha en hogares españoles en el año 2016 Fuente: Elaboración propia a partir de INE, 2016

Aunque resulte un tanto equívoco, lo cierto es que actualmente la conectividad se identifica con el acceso y prácticas de uso de Internet. Los datos relativos a esta dimensión aparecen representados en la gráfica de la Figura 2. De esta se desprende el espectacular crecimiento tecnológico experimentado en España, dato que surge al compararlos con la media europea. Si bien 
Waliño-Guerrero, Ma José; Pardo Baldoví, M. Isabel; Esnaola Horacek, Graciela y San Martín Alonso, Ángel (2018). "La participación escolar de las familias a través de plataformas digitales". @tic revista d'innovació educativa, 20, 80-88.

debemos matizar que en referencia a la Comunitat Valenciana, pese a que se alcanzan cifras de conectividad prácticamente iguales a la media europea, todavía queda situada por debajo de la media española (CECV, 2016). Esta desventaja se debe fundamentalmente a que dicho equipamiento tecnológico no es uniforme en el conjunto de la geografía de esta comunidad autónoma, de hecho, las tasas en sus amplias zonas rurales son significativamente inferiores a las urbanas. Dicho aspecto refleja la existencia de una brecha digital geográfica y, por tanto, las desigualdades se mantienen en torno al acceso a la tecnología. Estas diversas y preocupantes discontinuidades aparecen en la configuración de la ciudadanía digital (Rendueles, 2016).

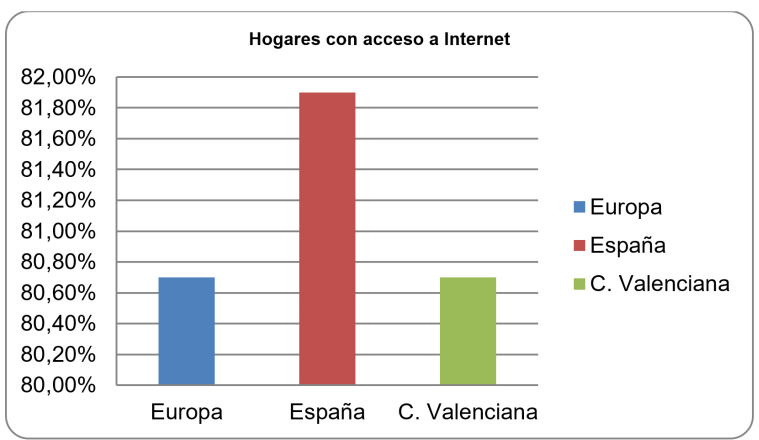

Figura 2. Gráfico del porcentaje de hogares con acceso a Internet en el año 2016, a nivel europeo, nacional (España) y autonómico (Comunitat Valenciana). Fuente: Elaboración propia a partir de ONTSI, 2016

Por lo que se refiere a la disponibilidad de equipos en los hogares, como se puede comprobar en la Figura 3, el equipamiento en la Comunitat Valenciana es bastante semejante al de la media del resto de las comunidades autónomas españolas. Si bien solo en móviles y televisores supera a la media de referencia, en todos los demás equipos alcanza tasas ligeramente inferiores.

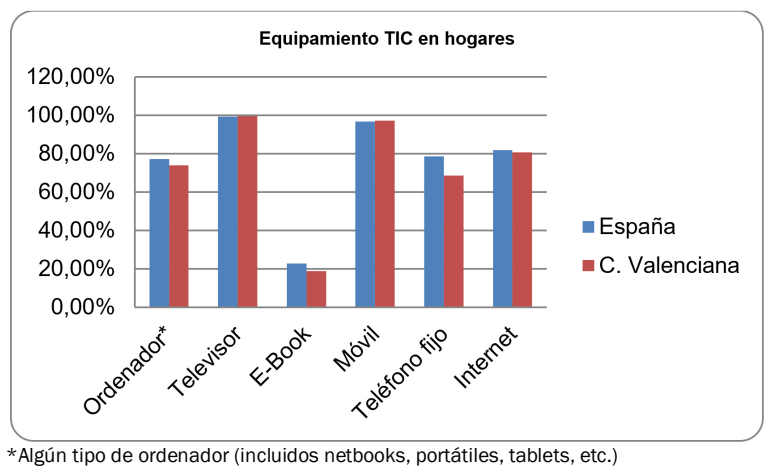

Figura 3. Gráfico del equipamiento TIC en hogares, año 2016, en España y la Comunitat Valenciana. Fuente: Elaboración propia a partir de INE, 2016

Es cierto que tanto la conectividad como el equipamiento de los hogares se adquieren para utilizarlos en un amplio espectro de tareas y servicios, tal como de alguna manera se representa en el gráfico de la Figura 4, y no únicamente para destinarlos a aspectos relacionados con el sistema escolar. No obstante, Ilama la atención que la Comunitat Valenciana está ligeramente por encima de la media del Estado en todas las categorías de tareas establecidas, incluso la diferencia es mayor en las más relacionadas con la participación en los asuntos escolares. Pero este detalle no debe sacarse de contexto, pues se trata de tareas en su conjunto muy semejantes al patrón de uso social de las tecnologías pautado por el mercado.

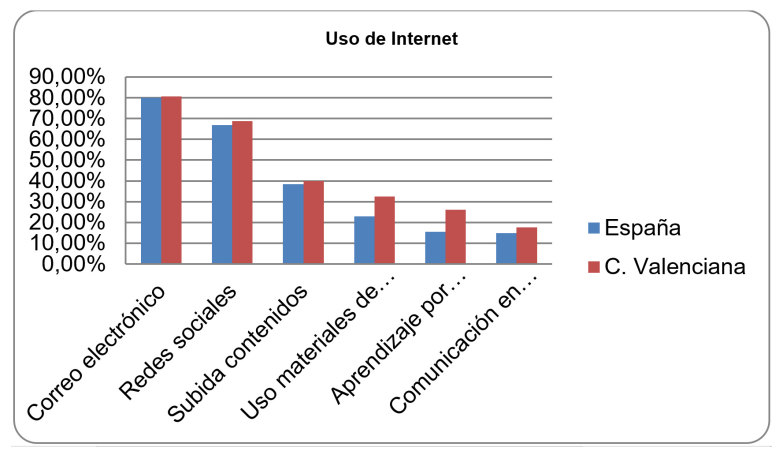

Figura 4. Gráfico del uso de Internet en el año 2016, en España y la Comunitat Valenciana. Fuente: Elaboración propia a partir de ONTSI, 2016

\subsection{Configuración del discurso sobre la participación}

Tras esta aproximación a la realidad que nos dibujan los datos recabados, y que nos han permitido reconstruir el perfil de conectividad de los hogares valencianos, ahora nos adentraremos en lo requerido por el segundo objetivo específico que son las categorías que describen la experiencia de participación de las familias entrevistadas en nuestro trabajo de campo.

Es fundamental, desde luego, indagar sobre la disponibilidad de los recursos tecnológicos como requisito para la participación de las familias en la escuela de la sociedad 2.0. Es aún más importante profundizar en la tipología de usos y prácticas que las familias hacen de tales artefactos en la relación participativa que establecen con el centro escolar. Tal como hemos mencionado más arriba, el análisis se ha realizado a partir de las categorías identificadas en las explicaciones y comentarios de las familias entrevistadas. A tal efecto realizamos un análisis de frecuencias, cuyos resultados sintetizamos en la Figura 5.

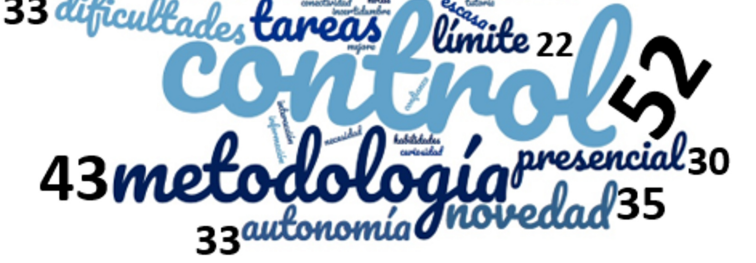

Figura 5. Nube de categorías en relación a la percepción de la participación de las familias según frecuencia. Fuente: Elaboración propia

En la figura precedente se observa que la pluralidad de categorías que aparecen pueden ser clasificadas en tres 
Waliño-Guerrero, Ma José; Pardo Baldoví, M. Isabel; Esnaola Horacek, Graciela y San Martín Alonso, Ángel (2018). "La participación escolar de las familias a través de plataformas digitales". @tic revista d'innovació educativa, 20, 80-88.

grandes grupos. El primero de ellos alude a los aspectos más tradicionales en relación a la participación, más vinculados con la interacción familia-escuela, como son los conceptos de: comunicación, participación, interacción, implicación, tutoría, confianza, autonomía, tradicionales o presencial. Por otro lado, se observa un segundo grupo relativo a los elementos propios del proceso de enseñanza-aprendizaje, en el que encontramos los términos de: motivación, metodología, tareas, habilidades, curiosidad, mejora o notas. Finalmente, se observa un tercer grupo de términos estrechamente relacionados con el ejercicio de la participación a través de las plataformas digitales y las dudas que esta suscita, como son: conectividad, control, límite, dificultades, incertidumbre, novedad o necesidad. Si nos centramos en las frecuencias de los términos, resulta significativo que el concepto más destacado por las familias sea el de control, lo que muestra cierta distorsión o desviación en cuanto a cómo entienden la participación. Es decir, la asociación de ambos conceptos (control y participación) sugiere que se tiende a conceder una orientación negativa o restringida a la participación, limitándola a la supervisión del proceso de enseñanza-aprendizaje o de la vida de la escuela, pero no entendiéndola como una participación efectiva y en sentido extenso.

No obstante, cabe enfatizar que esta supremacía del término control no se debe exclusivamente a la actitud de las familias, tal como hemos corroborado en las entrevistas y grupo de discusión. De ellas se desprende que es precisamente el ejercicio de la participación a través de las tecnologías y de las plataformas digitales lo que potencia, y en cierta medida llega a imponer, este nuevo modelo de participación. El trabajo mediante plataformas motiva un tipo distinto de implicación y respuesta de los participantes, que en un primer momento puede parecer más directa (ya que se produce en muchas ocasiones de manera instantánea y continua), pero que en realidad es más distante e impersonal, al ser ejercida por vía telemática y no de manera personal o presencial.

De lo anterior se deduce que la tecnología permite únicamente determinados tipos de interacción, así como determinados grados de implicación; a la vez que impide o dificulta otros, por lo que modula los procesos y estrategias de participación de las familias en la escuela. Este aspecto se vislumbra claramente en las entrevistas, en las cuales las familias coinciden en que los nuevos medios digitales provocan un desplazamiento constante de sus posibilidades y límites de actuación, relegando su papel a un segundo plano respecto al que ocupaban cuando se trabajaba en formato papel y se ejercía la participación de manera presencial.

Es decir, las entrevistas reflejan que actualmente las familias perciben que se ha producido un cambio de su rol en la escuela, de modo que ahora se conciben más como acompañantes que como verdaderos implicados o partícipes activos en el proceso. Afirmando este aspecto subrayamos las siguientes intervenciones de dos de los agentes entrevistados:

Ella tiene sus tareas claras. De hecho, en la tablet aparece todo lo que tiene que hacer... Además, cada vez que se cuelga algo nuevo y que los chicos han de hacer, la profesora manda un mensaje y mi hija sabe ya lo que tiene que hacer. (EFCS1: 698-700).
En sus tareas no podemos meternos, vemos que van solos y saben lo que tiene que hacer en cada momento... Lo único es que en casa les ponemos un límite, y es que, a la hora de dormir, tanto la tablet como el móvil se apagan y se quedan fuera de la habitación. (EFCS2: 564-565).

Ambas intervenciones reflejan un cambio de rol y de mentalidad en las familias, las cuales ahora se limitan a contemplar el trabajo del alumnado y a supervisar que no se produzcan desviaciones en el proceso. Quedando así lejos de las reivindicadas demandas de una participación activa y real de los agentes escolares con las instituciones educativas. Lo cual, sin duda, estaría introduciendo cambios relevantes en la gobernanza de las organizaciones y de la sociedad, cambios a los que convendría prestar mayor atención. (Dal Flore, 2007).

Siguiendo con el cambio del modelo de participación, vemos que otro de los conceptos destacados en las entrevistas es el de comunicación. En torno a este término cabe destacar que sucede algo muy similar a lo comentado anteriormente en relación a la limitación del concepto, y a su asociación con el concepto de supervisión. Ya que muchas familias indican que las plataformas digitales han permitido aumentar la comunicación entre las familias y el centro escolar. No obstante, cuando se les cuestiona en torno a los elementos en los que se ha producido dicho aumento de comunicación, suelen aludir solamente al intercambio de información sobre notas y tareas. En consecuencia, advertimos que ese pretendido aumento de comunicación es en realidad muy superficial y queda acotado únicamente a aquellos aspectos más técnicos o poco significativos. A modo de ilustración destacamos el siguiente fragmento de una entrevista a una madre que manifiesta mantener una excelente comunicación con el centro:

La maestra siempre ha tenido mucho interés en mantenernos bien informados, ya sea a través de la plataforma, del móvil, del Gmail o de reuniones con ella porque le gusta combinar lo presencia con lo virtual... Nos informa de cómo va mi hija, pero también de si van consiguiendo o no los objetivos del curso, las actividades que hacen, etc. (EFCS1: 738-740).

En este sentido también surgen voces críticas entre las familias que visibilizan algunas de las dificultades y problemáticas del ejercicio de la participación a través de las plataformas. Los entrevistados explicitan la disyuntiva entre cantidad versus calidad de la información. Manifiestan que el notable incremento de nuevos canales de participación (Figura 6), dispersa su atención y, sin embargo, perciben cierta falta de información respecto a aspectos fundamentales del proceso educativo. Y la necesidad de seguir comunicándose mediante los elementos y vías tradicionales (apuntan principalmente la tutoría presencial, la agenda en papel o la telefonía) para conseguir que se produzca verdaderamente una comunicación eficaz familia-escuela. 
Waliño-Guerrero, Ma José; Pardo Baldoví, M. Isabel; Esnaola Horacek, Graciela y San Martín Alonso, Ángel (2018). "La participación escolar de las familias a través de plataformas digitales". @tic revista d'innovació educativa, 20, 80-88.

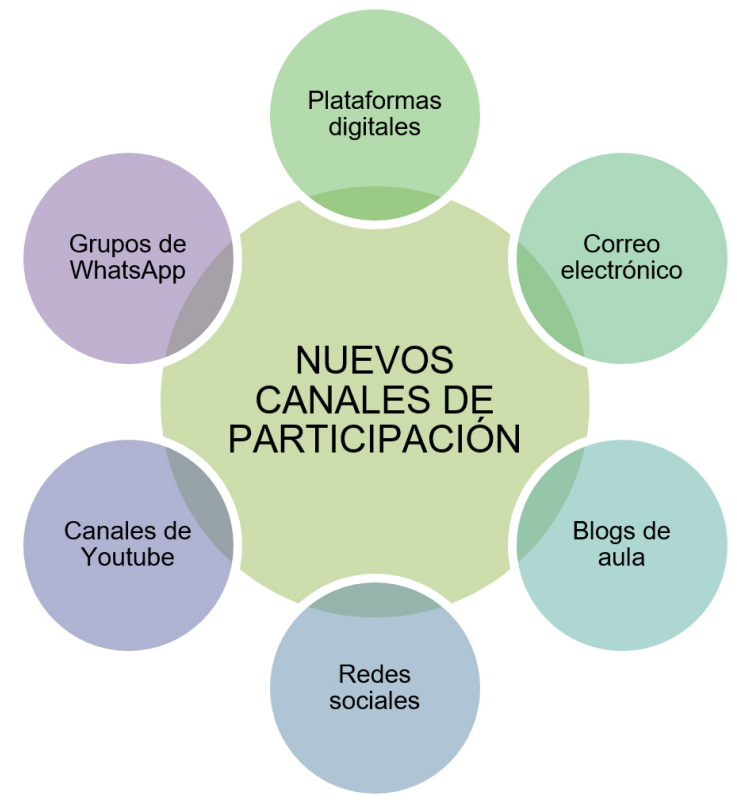

Figura 6. Nuevos canales de comunicación y participación familia-escuela. Fuente: Elaboración propia

Al hilo de esa cuestión, uno de los padres entrevistados nos decía respecto a la participación a través de plataformas digitales:

Ha empeorado la comunicación con el colegio, surgen dificultades de todo tipo, aunque sea mucho más ágil (...). Pienso que para estos temas tan delicados es mucho mejor el cara a cara. (EFCS3: 234-235).

En este fragmento en el que se evidencia otro de los aspectos destacados sobre todo en el grupo de discusión, que es precisamente las dudas suscitadas por el manejo de los dispositivos y de las plataformas digitales, lo que en muchas ocasiones actúa como una barrera para la participación de las familias, quienes ante el desconocimiento o los impedimentos de los problemas técnicos terminan por desistir de usar la plataforma digital y, por ende, de participar. Por tanto, se observa que en cierto modo el uso de las plataformas digitales no solo modula el sentido de la participación, sino que puede llegar incluso a coartarla o restringirla. Cabe plantearse si de este modo se puede fomentar esa escuela que concita y modula la pluralidad de valores sociales presentes en su entorno, tal como reclama Segovia (1986).

Cabe destacar, sobre todo, el consenso que muestran gran parte de las familias entrevistadas en torno a que las plataformas digitales utilizadas en la mayoría de los centros, tanto a nivel institucional como particular, no siempre facilitan la comunicación, sino que en ocasiones llegan a dificultarla. Ante esta situación se aducen dos razones: bien porque algunas familias todavía carecen de las habilidades necesarias para utilizar efectivamente estas herramientas tecnológicas; o bien por el problema de la conectividad al que aludíamos anteriormente, ya que algunos hogares todavía no disponen del equipamiento tecnológico o de conexión de banda ancha suficiente para acceder a estas plataformas de manera normalizada. En ocasiones también los nuevos canales son fuente de conflictos y malentendidos tanto entre las familias como entre éstas y la escuela, al ejercer la participación a distancia y no mediante procesos de interacción más directos.

El cruce de relaciones entre los términos manejados por nuestros informantes, quedan expuestos en el mapa relacional de conceptos de la Figura 7 . A partir de esta representación pueden observarse la frecuencia de las interacciones que se producen entre los términos manejados en los relatos formulados en las entrevistas y en el grupo focal.

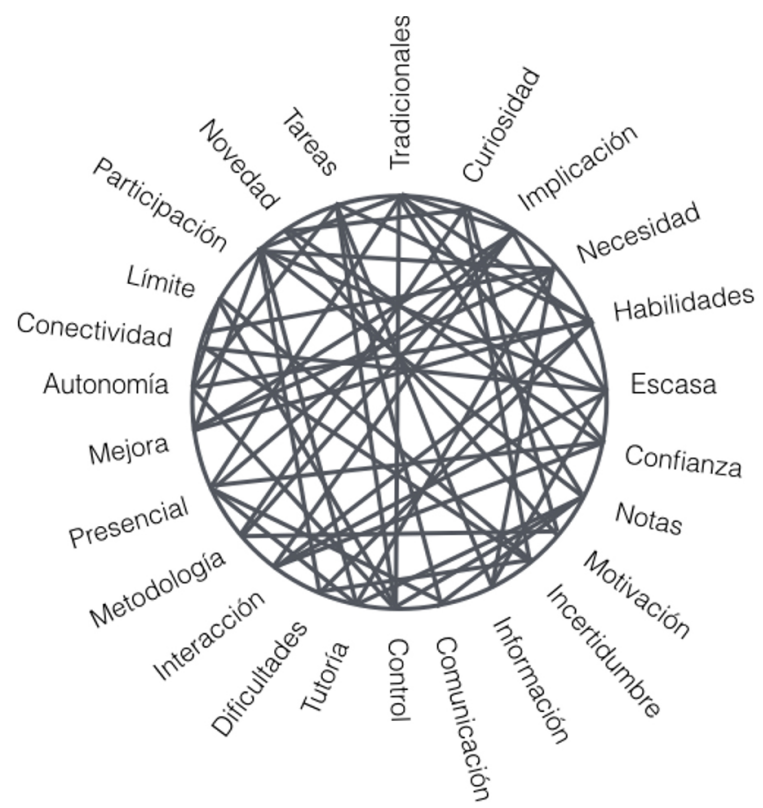

Figura 7. Mapa relacional sobre categorías de participación de las familias en la escuela. Fuente: Elaboración propia

Vemos en la Figura 7 que son muchas las categorías que los agentes escolares destacan en torno a las prácticas emergentes de participación mediante plataformas digitales. $Y$ todos ellos vienen a ratificar lo postulado por Jenkins (2008) en relación a que indudablemente se ha producido un cambio en la cultura participativa de la escuela. Un cambio promovido y posibilitado por la introducción de las tecnologías y por la normalización de las plataformas digitales como herramientas de gestión y de participación en la escuela. Y ante todo un cambio cuyas consecuencias y lógicas de acción escapan a la comprensión tanto de las familias como del resto de agentes implicados en la escuela, dado que no se basan exclusivamente en la acción aislada de los implicados, sino en la suma de las interacciones que se producen entre estos y en las sinergias e implicaciones derivadas de las mismas.

\section{Consideraciones finales}

En el encabezamiento de este texto aparecía una frase contundente del sociólogo De Sousa pidiendo la desmercantilización de determinados ámbitos de nuestra vida colectiva. Sin duda, uno de ellos debería ser el de todo aquello relacionado con las estrategias que regulan la convivencia, como lo es la participación. No es suficiente con hablar de ella, pues como dice Pisani (2015) es una de las palabras con mayor presencia en los discursos políticos, mucho más en los electorales, pero casi nadie precisa cómo llevar a cabo realmente la participación. Tal vez porque hay muchas fórmulas para 
Waliño-Guerrero, Ma José; Pardo Baldoví, M. Isabel; Esnaola Horacek, Graciela y San Martín Alonso, Ángel (2018). "La participación escolar de las familias a través de plataformas digitales". @tic revista d'innovació educativa, 20, 80-88.

ello, y una de estas en fase de experimentación es la que adoptan como instrumento de mediación los medios y redes digitales, tal como tratamos de sugerir con las escenas al inicio de este texto.

De hecho, los agentes entrevistados están ya en esta fase de experimentación a partir de su equipamiento privado y su participación cotidiana en redes sociales, poniendo de manifiesto una serie de cuestiones sobre las que hemos de reflexionar. Una de ellas es que no hay una transferencia propiamente dicha entre las prácticas de uso con los medios tecnológicos y las formas tradicionales de intervenir en los procesos de toma de decisiones en los ámbitos educativos. La implicación instrumental no se traslada a la política, salvo en fomentar la deriva de la participación hacia los derechos individuales y no tanto a los colectivos (Feito, 2014). Por otra parte, esa implicación se focaliza en mayor medida hacia las dimensiones de control jerárquico sobre las tareas y procesos, y en menor medida en formar parte en las estrategias de desarrollo organizacional. Déficit que convendría revisar puesto que nuestros informantes interactúan en plataformas que facilitan contenidos digitales de carácter formativo.

Se ha de prestar especial atención a las discontinuidades, como señala Rendueles (2016), en diferentes ámbitos. Uno de ellos es la distancia que separa a los estudiantes de la pública y la privada, pues, aunque los centros de este sector están peor equipados, los hogares disponen de mejor conectividad para interactuar con las plataformas de contenidos. Por otro lado, los usuarios y usuarias no diferencian entre las plataformas que les facilita la administración pública de las que son estrictamente privadas, aunque admiten que estas funcionan mejor que las otras sin advertir la presencia mercantil en las actividades de participación.

Una cuestión que hemos apuntado a lo largo de este trabajo y sobre la que no hemos reparado lo suficiente, al menos en cuanto a su configuración empírica, es a las diferencias arraigadas en esta cuestión importante en toda democracia. Se trata de diferencias que responden a la condición de género, de disponibilidad de recursos económicos o de la zona geográfica en la que habiten las familias. Por lo que nuestro propósito es continuar indagando, pero considerando tales diferencias y sobre todo en los entornos rurales y otros igualmente desfavorecidos de la Comunitat Valenciana para poder abrir el enfoque del estudio a otras realidades.

Del mismo modo, también consideramos interesante realizar un estudio más intensivo del nuevo modelo de participación en distintos contextos y niveles educativos, especialmente en el universitario. Por ser este uno de los núcleos en los que más se intensifica la participación a través de las redes y las tecnologías, tal y como se vislumbra en el presente artículo. A la luz de lo expuesto queda patente que la participación de los agentes educativos a través de las tecnologías, con las luces y sombras asociadas a este nuevo modelo, abre un amplio abanico de oportunidades de estudio e investigación que pueden resultar de gran interés, y en los cuales pretendemos continuar explorando.

\section{Bibliografía}

Aparici, R. y Osuna Acedo, S. (2013). La cultura de la participación. Revista Mediterránea de comunicación, 4(2), 137-148. https://doi.org/10.14198/MEDCOM2013.4.2.07
Arendt, H. (1996). Entre el pasado y el futuro. Barcelona: Península.

Bates, (1989). Burocracia, educación y democracia: Hacia una política de participación 190-205. En Bates, R. (Ed.). Práctica crítica de la administración educativa. Valencia: Servei de Publicacions Universitat de València.

Ball, S. (2016). Gobernanza neoliberal y democracia patológica. 23-40. En Collet y Tort (Coords.). La gobernanza escolar democrática. Madrid: Morata.

Benkler, Y. (2015). La riqueza de las redes. Barcelona: Icaria.

Boudon, R. (1983). La desigualdad de oportunidades. Barcelona: Editorial Laia.

CECV, (2016). Informe sobre la situación del Sistema Educativo en la Comunidad Valenciana. Cursos 2013/14 y 2014/15. Recuperado de: https://goo.gl/Td5zSF

Dal Flore, F. (2007). Communities Versus Networks. The Implications on Innovation and Social Change. American Behavioral Scientist, 50(7), 857-866. https://doi.org/10.1177/0002764206298311

De Sousa, B. (2016): La difícil democracia. Una mirada desde la periferia europea. Madrid: Akal.

European Commission (2015). Survey of Schools: ICT in Education. Spain. Bruxelles: European Schoolnet.

Feito, R. (2014). Treinta años de consejos escolares. La participación de los padres y de las madres en el control y gestión de los centros sostenidos con fundo públicos en España. Profesorado. Revista de currículum y formación del profesorado. 18(2), 5167.

Freeman, A., Adams Becker, S., Cummins, M., Davis, A., y Hall Giesinger, C. (2017). NMC/COSN (2017). Horizon Report 2017 K-12 Edition. Recuperado de: https://cdn.nmc.org/media/2017-nmc-cosn-horizonreport-k12-EN.pdf

Gill, G. (2011). Informing with the Case Method. New York: Informing Science Press.

Gómez, L. (2000). Educación pública. Madrid: Morata.

Helsper, E. J. y Eynon, R. (2010). Digital natives: where is the evidence? British Educational Research Journal, 36 (3), 503-520.

INE. (2016). Encuesta sobre Equipamiento y Uso de Tecnologías de Información y Comunicación en los Hogares. Año 2016. Recuperado de: http://www.ine.es/dyngs/INEbase/es/operacion.htm ?c=Estadistica_C\&cid=1254736176741\&menu=ulti Datos\&idp $=1254735976608$

Inglehart, R. y Welzel, Ch. (2006). Modernización, cambio cultural y democracia: la secuencia del desarrollo humano. Madrid: CIS-Siglo XXI.

Innerarity, D. (2011). La política después de la indignación. Claves de razón práctica, 218, 30-42.

Jenkins, H. (2008). Convergence Culture. Where Old and New Media Collide. New York: New York University Press.

https://doi.org/10.1177/1354856507084415 
Waliño-Guerrero, Ma José; Pardo Baldoví, M. Isabel; Esnaola Horacek, Graciela y San Martín Alonso, Ángel (2018). "La participación escolar de las familias a través de plataformas digitales". @tic revista d'innovació educativa, 20, 80-88.

Macià, M. (2016). La comunicación familia-escuela: el uso de las TIC en los centros de primaria. Revista Electrónica Interuniversitaria de Formación del Profesorado, 19(1), 73-83.

Mattelart, A. y Vitalis, A. (2015). De Orwell al cibercontrol. Barcelona: Gedisa.

Maxcy, S. J. (1995). Democracy, Chaos, and the New School Order. London: Corwin Press.

ONTSI (2017). La sociedad en red. Informe anual 2016. Recuperado http://www.ontsi.red.es/ontsi/es/content/informeanual-la-sociedad-en-red-2016-edici\%C3\%B3n-2017

Pereira, Z. (2011). Los diseños de método mixto en la investigación en educación. Una experiencia concreta. Educare, 15(1), 15-29.

Pérez, F. y Uriel, E. (2016). Cuentas de la educación en España, 2000-2013. Valencia: IVIE-Fundación BBVA.

Pisani, F. (2015). Facilitar la participación ciudadana. Recuperado de: http://www.francispisani.net/2015/06/facilitar-laparticipacion-ciudadana.html

Rendueles, C. (2016). La ciudadanía digital. ¿Ágora aumentada 0 individualismo postmaterialista? Revista Latinoamericana de Tecnología Educativa,15(2), 15-24.

San Martín, A. y Muñoz, J. L. (2015). La participación como estrategia de intermediación en las organizaciones escolares. Organización y gestión educativa, 23(1), 16-19.

Sartori, G. (1998). Homo videns. La sociedad teledirigida. Madrid: Taurus.

Segovia Pérez, J. (1986). Participación en la educación: vivencia democrática. Revista de Estudios de Juventud, 23, 17-21.

Sennett, R. (2000). La corrosión del carácter. Barcelona: Anagrama.

Sennett, R. (2006). La cultura del nuevo capitalismo. Barcelona: Anagrama.

Shields, R. Ed. (1996). Cultures of internet. Virtual Spaces, Real Histories, Living Bodies. London: Sage Publications.
Smith, J., Wohlstetter, P., Kuzin, C. A., y De Pedro, K. (2011). Parent Involvement in Urban Charter Schools: New Strategies for Increasing Participation. School Community Journal, 21(1), 71-94.

Stake, R. (2010). Investigación con estudio de casos. Madrid: Morata.

Subirats, J. (2016). Notas sobre principios y estrategias de una gobernanza educativa y democrática de lo común en el cambio de época. En Collet y Tort (Coords.). La gobernanza escolar democrática. Madrid: Morata.

Telefónica (2018). Sociedad digital en España-2017. Madrid: Fundación Telefónica-Ariel.

Vanvergen, P. (1977). Escuela y comunidad. Revista de educación, 252, 54-56.

VV. AA. (2015). El proyecto de banda ancha ultrarrápida para los centros escolares españoles. Madrid: Red.es.

Zhao, Sh., Grasmuck, S. y Martin, J. (2008). Identity construction on Facebook: digital empowerment in anchored relationships. Computers in human behavior, vol. 24, 1816-1836. https://doi.org/10.1016/j.chb.2008.02.012.

| Cita recomendada de este artículo

Waliño-Guerrero, Ma José; Pardo Baldoví, M. Isabel; Esnaola Horacek, Graciela y San Martín Alonso, Ángel (2018). “La participación escolar de las familias a través de plataformas digitales". @tic revista d'innovació educativa, 20, 80-88. 